\title{
RELATIONS OF COMBINATORIC CONVOLUTION SUMS FOR RESTRICTED DIVISOR FUNCTIONS AND BERNOULLI POLYNOMIALS
}

\author{
DAEYEOUL KIM AND NAZLI YILDIZ IKIKARDES
}

Received 03 June, 2020

\begin{abstract}
In this paper, we study combinatoric convolution sums involving divisor functions. First, we establish some explicit formulas for certain combinatoric convolution sums of divisor functions derived from Bernoulli polynomials. Second, we show a formula of the fourth order of convolution sums of divisor functions expressed by their divisor functions and linear combination of Bernoulli polynomials.
\end{abstract}

2010 Mathematics Subject Classification: 11A05, 33E99

Keywords: Bernoulli Polynomials, Convolution sums, Divisor functions, Linear combination of polynomials

\section{INTRODUCTION AND STATEMENT OF MAIN RESULTS}

The symbols $\mathbb{N}, \mathbb{Z}$ and $\mathbb{R}$ denote the set of natural numbers, the ring of integers and the field of real numbers, respectively. Over the years Bernoulli polynomials have been used to prove important mathematical theorems. Since the 17th century, many mathematicians in different fields have been interested in Bernoulli polynomials. The Bernoulli polynomials $B_{k}(x)$, which are usually defined by the exponential generating function

$$
\frac{t e^{x t}}{e^{t}-1}=\sum_{k=0}^{\infty} \frac{B_{k}(x)}{k !} t^{k}
$$

play an important and quite mysterious role in mathematics and various fields like analysis, number theory and differential topology. The Bernoulli numbers $B_{k}$ are defined to be $B_{k}:=B_{k}(0)$.

The Bernoulli polynomial [7] is expressed through the respective numbers

$$
B_{n}(x)=\sum_{k=0}^{n}\left(\begin{array}{l}
n \\
k
\end{array}\right) B_{k} x^{n-k}
$$

* This work was supported by The Research Fund of Balikesir University, Project No: 2014/55. 


$$
\begin{aligned}
& B_{n}(x+y)=\sum_{k=0}^{n}\left(\begin{array}{l}
n \\
k
\end{array}\right) B_{k}(x) y^{n-k}, \\
& B_{n}^{\prime}(x)=n B_{n-1}(x) \\
& B_{n}(1-x)=(-1)^{n} B_{n}(x) \text { and } \\
& B_{n}(1+x)-B_{n}(x)=n x^{n-1} .
\end{aligned}
$$

For $n \in \mathbb{N}$ and $k \in \mathbb{Z}$, we define two divisor functions

$$
\sigma_{k}(n):=\sum_{d \mid n} d^{k}, \quad \hat{\sigma}_{k}(n):=\sum_{d \mid n}(-1)^{\frac{n}{d}-1} d^{k}, \quad \sigma_{k}^{*}(n):=\sum_{\substack{d \mid n \\ \frac{n}{d} \text { odd }}} d^{k} .
$$

The identity

$$
\sum_{k=1}^{n-1} \sigma(k) \sigma(n-k)=\frac{5}{12} \sigma_{3}(n)+\left(\frac{1}{12}-\frac{1}{2} n\right) \sigma(n)
$$

for the basic convolution sum first appeared in a letter from Besge to Liouville in 1862 ([3]). Hahn [6, (4.8)] considered

$$
36 \sum_{m<n} \hat{\boldsymbol{\sigma}}(m) \hat{\boldsymbol{\sigma}}(n-m)= \begin{cases}-3 \hat{\sigma}(n)+3 \widetilde{\sigma}_{3}(n), & \text { if } n \text { is odd, } \\ -3 \hat{\sigma}(n)-5 \widetilde{\sigma}_{3}(n)+4 \widetilde{\sigma}_{3}\left(\frac{n}{2}\right), & \text { if } n \text { is even. }\end{cases}
$$

It is well-known that $\hat{\sigma}_{k}(n)=\sigma_{k}(n)-2 \sigma_{k}\left(\frac{n}{2}\right)([6,(1.13)])$.

In this article, we are trying to focus on the combinatorial convolution sums. For positive integers $k, l, n, p$ and $q$ the combinatorial convolution sums

$$
\sum_{s=0}^{k-1}\left(\begin{array}{c}
2 k \\
2 s+1
\end{array}\right) \sum_{m=1}^{n-1} \hat{\sigma}_{2 k-2 s-1}(m) \hat{\sigma}_{2 s+1}(n-m)
$$

and

$$
\sum_{\substack{1 \leq m \leq p-1 \\
1 \leq m^{\prime} \leq q-1 \\
a, b, c, d \text { odd } \\
a+b+c+d=2 l}}\left(\begin{array}{c}
2 l \\
a, b, c, d
\end{array}\right) \hat{\sigma}_{a}(m) \hat{\sigma}_{b}(p-m) \hat{\sigma}_{c}\left(m^{\prime}\right) \hat{\sigma}_{d}\left(q-m^{\prime}\right)
$$

can be evaluated explicitly in terms of divisor functions and a sum involving Bernoulli polynomials.

We are motivated by Ramanujan's recursion formula for sums of the product of two Eisenstein series [2] and its proof, and also the following identities ([1],[4],[9]):

$$
\begin{aligned}
\sum_{s=0}^{k-1}\left(\begin{array}{c}
2 k \\
2 s+1
\end{array}\right) & \sum_{m=1}^{N-1} \sigma_{2 k-2 s-1}(m) \sigma_{2 s+1}(N-m) \\
& =\frac{2 k+3}{4 k+2} \sigma_{2 k+1}(N)+\left(\frac{k}{6}-N\right) \sigma_{2 k-1}(N)
\end{aligned}
$$




$$
+\frac{1}{2 k+1} \sum_{j=2}^{k}\left(\begin{array}{c}
2 k+1 \\
2 j
\end{array}\right) B_{2 j} \sigma_{2 k+1-2 j}(N) .
$$

Using these new formulas and addition theorem of Bernoulli polynomials, we derive the explicit formulas for quadnomial convolution sums of divisor functions. We define $\hat{B}_{2 k+1}(n)$ that if $n$ is even, then $\sum_{d \mid n} B_{2 k+1}(d)-2 \sum_{d \mid \frac{n}{2}} B_{2 k+1}(d)$ and if $n$ is odd, then $\sum_{d \mid n} B_{2 k+1}(d)$. Similarly, $\hat{\hat{B}}_{2 k+1}(n)$ that if $n$ is even, then $\sum_{d \mid n} \hat{B}_{2 k+1}(d)-$ $2 \sum_{d \mid \frac{n}{2}} \hat{B}_{2 k+1}(d)$ and if $n$ is odd, then $\sum_{d \mid n} \hat{B}_{2 k+1}(d)$. More precisely, we prove the following results.

Theorem 1. If $k \geq 1$ and $n \geq 2$, then

$$
\begin{aligned}
& \sum_{s=0}^{k-1}\left(\begin{array}{c}
2 k \\
2 s+1
\end{array}\right) \sum_{m=1}^{n-1} \hat{\sigma}_{2 k-2 s-1}(m) \hat{\sigma}_{2 s+1}(n-m) \\
& =\frac{1}{2} \sigma_{2 k+1}(n)-\frac{1}{2} \hat{\sigma}_{2 k}(n)-\frac{1}{2 k+1} \hat{B}_{2 k+1}(n) .
\end{aligned}
$$

Theorem 2. If $k \geq 2$ and $N \geq 1$, then

$$
\begin{aligned}
& \sum_{s=1}^{k-1}\left(\begin{array}{c}
2 k \\
2 s+1
\end{array}\right) \sum_{m=1}^{2 N-1}(-1)^{m} \hat{\sigma}_{2 k-2 s-1}(m) \hat{\sigma}_{2 s+1}(2 N-m) \\
& =\frac{1}{2} \sigma_{2 k+1}(N)-\frac{1}{2} \hat{\sigma}_{2 k}(2 N)-\frac{1}{2 k+1} \hat{B}_{2 k+1}(2 N) .
\end{aligned}
$$

Corollary 1. For $N \geq 1$, we have

$$
\begin{aligned}
& \sum_{\substack{m=1 \\
m \text { odd } \\
l \text { even }}}^{2 N-1} \hat{\sigma}_{l-1}(m) \hat{\boldsymbol{\sigma}}(2 N-m) \\
& \quad=\frac{1}{2 l}\left[\sigma_{l+1}(N)+\sigma_{l+1}(2 N)\right]-\frac{1}{l} \hat{\sigma}_{l}(2 N)-\frac{2}{l(l+1)} \hat{B}_{l+1}(2 N) .
\end{aligned}
$$

Corollary 2. For $k \geq 2$ and $N \geq 1$, we have

$$
\begin{aligned}
& \sum_{s=1}^{k-1}\left(\begin{array}{c}
2 k \\
2 s+1
\end{array}\right) \sum_{\substack{m=1 \\
m \text { odd }}}^{2 N-1} \hat{\sigma}_{2 k-2 s-1}(m) \hat{\sigma}_{2 s+1}(2 N-m)=2^{2 k-1} \sigma_{2 k+1}^{*}(N) \\
& \quad-\frac{1}{4}\left[\sigma_{2 k+1}(N)+\sigma_{2 k+1}(2 N)\right]-\frac{1}{2} \hat{\sigma}_{2 k}(2 N)+\frac{1}{2 k+1} \hat{B}_{2 k+1}(2 N) .
\end{aligned}
$$

Theorem 3. Let $m \in \mathbb{N}, m \geq 1$ and $x, y \in \mathbb{R}$. Then we have

$$
\begin{array}{r}
\sum_{k=1}^{m-1}\left(\begin{array}{c}
2 m \\
2 k
\end{array}\right) \frac{B_{2 k+1}(x) B_{2 m-2 k+1}(y)}{(2 k+1)(2 m-2 k+1)}=\frac{1}{4 m+4}\left\{B_{2 m+2}(y-x)\right. \\
\left.-B_{2 m+2}(x+y)\right\}-\frac{1}{2 m+1}\left\{x B_{2 m+1}(y)+y B_{2 m+1}(x)\right\}
\end{array}
$$




$$
\begin{aligned}
& +\frac{1}{4 m+2}\left\{B_{2 m+1}(x)+B_{2 m+1}(y)+(x+y-1) B_{2 m+1}(x+y)\right. \\
& \left.\quad+(x-y) B_{2 m+1}(y-x)\right\} .
\end{aligned}
$$

The following theorem gives a formula of the fourth order of convolution sums of divisor functions expressed by their divisor functions and linear combination of Bernoulli polynomials.

Theorem 4. Let $l, p, q \in \mathbb{N}$ with greater than 1 . Then

$$
\begin{aligned}
& \sum_{\substack{1 \leq m \leq p-1 \\
1 \leq m^{\prime} \leq q-1}}\left(\begin{array}{c}
2 l \\
a, b, c, d
\end{array}\right) \hat{\boldsymbol{\sigma}}_{a}(m) \hat{\boldsymbol{\sigma}}_{b}(p-m) \hat{\boldsymbol{\sigma}}_{c}\left(m^{\prime}\right) \hat{\boldsymbol{\sigma}}_{d}\left(q-m^{\prime}\right) \\
& a, b, c, d \text { odd } \\
& a+b+c+d=2 l \\
& =\frac{1}{8(2 l+1)}\left\{\hat{\hat{B}}_{2 l+1}(q+p+1)+\hat{\hat{B}}_{2 l+1}(q-p+1)-\hat{\hat{B}}_{2 l+1}(q+p)-\hat{\hat{B}}_{2 l+1}(q-p)\right. \\
& +\sum_{\substack{d\left|p \\
d^{\prime}\right| q}} d^{\prime} d\left[B_{2 l+1}\left(d^{\prime}+d+1\right)+B_{2 l+1}\left(d^{\prime}-d+1\right)-B_{2 l+1}\left(d^{\prime}+d\right)-B_{2 l+1}\left(d^{\prime}-d\right)\right] \\
& -\sum_{d \mid p} d\left[\hat{B}_{2 l+1}(q+d+1)+\hat{B}_{2 l+1}(q-d+1)-\hat{B}_{2 l+1}(q+d)-\hat{B}_{2 l+1}(q-d)\right] \\
& \left.-\sum_{d^{\prime} \mid q} d^{\prime}\left[\hat{B}_{2 l+1}\left(d^{\prime}+p+1\right)+\hat{B}_{2 l+1}\left(d^{\prime}-p+1\right)-\hat{B}_{2 l+1}\left(d^{\prime}+p\right)-\hat{B}_{2 l+1}\left(d^{\prime}-p\right)\right]\right\} \\
& +\frac{1}{4(2 l+1)}\left\{2 \hat{\hat{B}}_{2 l+1}(p+q)+\hat{\hat{B}}_{2 l+1}(p-q)+\hat{\hat{B}}_{2 l+1}(q-p)\right. \\
& \left.-\sum_{d \mid p} d\left[\hat{B}_{2 l+1}(q+d)+\hat{B}_{2 l+1}(q-d)\right]-\sum_{d^{\prime} \mid q} d^{\prime}\left[\hat{B}_{2 l+1}\left(p+d^{\prime}\right)+\hat{B}_{2 l+1}\left(p-d^{\prime}\right)\right]\right\} \\
& +\frac{1}{2(2 l+1)}\left\{(p+q-1) \hat{\hat{B}}_{2 l+1}(p+q)+(p-q) \hat{\hat{B}}_{2 l+1}(q-p)\right. \\
& -(2 p-1) \hat{\hat{B}}_{2 l+1}(q)-(2 q-1) \hat{\hat{B}}_{2 l+1}(p) \\
& +\hat{B}_{2 l+1}(p)\left[\sigma_{1}(q)-\sigma_{0}(q)+2 \sigma_{0}\left(\frac{q}{2}\right)\right] \\
& \left.+\hat{B}_{2 l+1}(q)\left[\sigma_{1}(p)-\sigma_{0}(p)+2 \sigma_{0}\left(\frac{p}{2}\right)\right]\right\} \\
& +\left[\frac{1}{4} \sigma_{1}(p)-\sigma_{1}\left(\frac{p}{2}\right)\right]\left[\sigma_{2 l+1}(q)-\sigma_{2 l}(q)+2 \sigma_{2 l}\left(\frac{q}{2}\right)\right] \\
& +\left[\sigma_{2 l+1}(p)-\sigma_{2 l}(p)+2 \sigma_{2 l}\left(\frac{p}{2}\right)\right]\left[\frac{1}{4} \sigma_{1}(q)-\sigma_{1}\left(\frac{q}{2}\right)\right]
\end{aligned}
$$




$$
-\frac{1}{4(l+1)}\left[\hat{\hat{B}}_{2 l+2}(p+q)-\hat{\hat{B}}_{2 l+2}(q-p)\right] \text {. }
$$

\section{PROPERTIES OF COMBINATORIC CONVOLUTION SUMS OF DIVISOR FUNCTIONS DERIVED FROM BERNOULLI POLYNOMIALS}

The purpose of this section is to give proofs of the main results. Also, we would like to enrich this section with examples. To proof of the theorems, we need the auxiliary results and lemmas.

Lemma 1. ([5]) Let $k, n$ be positive integers. Then

$$
\begin{aligned}
\sum_{j=2}^{k}\left(\begin{array}{c}
2 k+1 \\
2 j
\end{array}\right) B_{2 j} \hat{\sigma}_{2 k+1-2 j}(n)=(2 k+1) \sigma_{2 k+1}^{*}(n) \\
\quad-\left(\frac{2 k+3}{2}\right) \hat{\sigma}_{2 k+1}(n)-\left(\frac{k(2 k+1)}{6}\right) \hat{\sigma}_{2 k-1}(n) \\
-(2 k+1) \sum_{s=0}^{k-1}\left(\begin{array}{c}
2 k \\
2 s+1
\end{array}\right) \sum_{m=1}^{n-1} \hat{\sigma}_{2 k-2 s-1}(m) \hat{\sigma}_{2 s+1}(n-m) .
\end{aligned}
$$

Lemma 2. ([8]) Let $m \in \mathbb{N}$ and $x, y \in \mathbb{R}$. Then we have

(a) $\sum_{k=0}^{m}\left(\begin{array}{l}m \\ k\end{array}\right) \frac{B_{k+1}(x)}{k+1} \frac{B_{m-k+1}(y)}{m-k+1}$

$$
=(x+y-1) \frac{B_{m+1}(x+y)}{m+1}-\frac{B_{m+2}(x+y)}{m+2}-\frac{B_{m+2}(x)}{(m+1)(m+2)}-\frac{B_{m+2}(y)}{(m+1)(m+2)} .
$$

(b) $\sum_{k=0}^{m}(-1)^{k}\left(\begin{array}{c}m \\ k\end{array}\right) \frac{B_{k+1}(x)}{k+1} \frac{B_{m-k+1}(y)}{m-k+1}$

$$
=(x-y) \frac{B_{m+1}(y-x)}{m+1}+\frac{B_{m+2}(y-x)}{m+2}+\frac{B_{m+2}(1-x)}{(m+1)(m+2)}+\frac{B_{m+2}(y)}{(m+1)(m+2)} .
$$

Now we are ready to prove the main results of the article.

Proof of the Theorem 1. Consider

$$
\begin{aligned}
T & :=\sum_{j=2}^{k}\left(\begin{array}{c}
2 k+1 \\
2 j
\end{array}\right) B_{2 j} \hat{\sigma}_{2 k+1-2 j}(n) \\
& =\sum_{j=0}^{2 k+1}\left(\begin{array}{c}
2 k+1 \\
j
\end{array}\right) B_{j} \hat{\boldsymbol{\sigma}}_{2 k+1-j}(n)-\sum_{j=0}^{k}\left(\begin{array}{c}
2 k+1 \\
2 j+1
\end{array}\right) B_{2 j+1} \hat{\sigma}_{2 k+1-(2 j+1)}(n)
\end{aligned}
$$




$$
-\left(\begin{array}{c}
2 k+1 \\
0
\end{array}\right) B_{0} \hat{\sigma}_{2 k+1}(n)-\left(\begin{array}{c}
2 k+1 \\
2
\end{array}\right) B_{2} \hat{\sigma}_{2 k+1-2}(n) .
$$

It is well-known that $B_{0}=1, B_{1}=-\frac{1}{2}, B_{2}=\frac{1}{6}$ and $B_{2 j+1}=0(j \geq 1)$. Thus

$$
\begin{aligned}
T= & \sum_{j=0}^{2 k+1}\left(\begin{array}{c}
2 k+1 \\
j
\end{array}\right) B_{j} \hat{\sigma}_{2 k+1-j}(n)-\left(\begin{array}{c}
2 k+1 \\
0
\end{array}\right) B_{0} \hat{\sigma}_{2 k+1}(n) \\
& \quad-\left(\begin{array}{c}
2 k+1 \\
1
\end{array}\right) B_{1} \hat{\sigma}_{2 k}(n)-\left(\begin{array}{c}
2 k+1 \\
2
\end{array}\right) B_{2} \hat{\sigma}_{2 k-1}(n) \\
= & \sum_{j=0}^{2 k+1}\left(\begin{array}{c}
2 k+1 \\
j
\end{array}\right) B_{j} \hat{\sigma}_{2 k+1-j}(n)-\hat{\sigma}_{2 k+1}(n)+\left(k+\frac{1}{2}\right) \hat{\sigma}_{2 k}(n)-\frac{k(2 k+1)}{6} \hat{\sigma}_{2 k-1}(n) .
\end{aligned}
$$

Using $B_{n}(x)=\sum_{l=0}^{n}\left(\begin{array}{c}n \\ l\end{array}\right) B_{l} x^{n-l}$, we obtain

$$
\begin{aligned}
\sum_{j=0}^{2 k+1}\left(\begin{array}{c}
2 k+1 \\
j
\end{array}\right) B_{j} & \left(\sigma_{2 k+1-j}(n)-2 \sigma_{2 k+1-j}\left(\frac{n}{2}\right)\right) \\
& =\sum_{j=0}^{2 k+1}\left(\begin{array}{c}
2 k+1 \\
j
\end{array}\right) B_{j}\left(\sum_{d \mid n} d^{2 k+1-j}-2 \sum_{d \mid \frac{n}{2}} d^{2 k+1-j}\right) \\
& =\sum_{d \mid n} B_{2 k+1}(d)-2 \sum_{d \mid \frac{n}{2}} B_{2 k+1}(d) \\
& =\hat{B}_{2 k+1}(n) .
\end{aligned}
$$

Combining (2.1), Lemma 1 and (2.2) we deduce that

$$
\begin{aligned}
& \sum_{j=0}^{2 k+1}\left(\begin{array}{c}
2 k+1 \\
j
\end{array}\right) B_{j} \hat{\sigma}_{2 k+1-j}(n)-\hat{\sigma}_{2 k+1}(n)+\left(k+\frac{1}{2}\right) \hat{\sigma}_{2 k}(n)-\frac{k(2 k+1)}{6} \hat{\sigma}_{2 k-1}(n) \\
& \quad=\hat{B}_{2 k+1}(n)-\hat{\sigma}_{2 k+1}(n)+\left(k+\frac{1}{2}\right) \hat{\sigma}_{2 k}(n)-\frac{k(2 k+1)}{6} \hat{\sigma}_{2 k-1}(n) \\
& \quad-(2 k+1) \sigma_{2 k+1}^{*}(n)+\left(\frac{2 k+3}{2}\right) \hat{\sigma}_{2 k+1}(n)+\left(\frac{k(2 k+1)}{6}\right) \hat{\sigma}_{2 k-1}(n) \\
& \quad=-(2 k+1) \sum_{s=0}^{k-1}\left(\begin{array}{c}
2 k \\
2 s+1
\end{array}\right) \sum_{m=1}^{n-1} \hat{\sigma}_{2 k-2 s-1}(m) \hat{\sigma}_{2 s+1}(n-m),
\end{aligned}
$$


and we obtain

$$
\begin{aligned}
& \sum_{s=0}^{k-1}\left(\begin{array}{c}
2 k \\
2 s+1
\end{array}\right) \sum_{m=1}^{n-1} \hat{\sigma}_{2 k-2 s-1}(m) \hat{\sigma}_{2 s+1}(n-m) \\
& =\frac{1}{2} \sigma_{2 k+1}(n)-\frac{1}{2} \hat{\sigma}_{2 k}(n)-\frac{1}{2 k+1} \hat{B}_{2 k+1}(n)
\end{aligned}
$$

Remark 1. With $k=1$ in Theorem 1, we recover (1.1).

Example 1. With $k=1,2,3$ in Theorem 1 look as

$$
\text { (a) } \begin{aligned}
\quad \hat{B}_{3}(n) & =\frac{3}{4} \sigma_{3}(n)-\frac{3}{2} \hat{\sigma}_{2}(n)-3 \sum_{m=1}^{n-1} \hat{\sigma}_{1}(m) \hat{\sigma}_{1}(n-m), \\
\text { (b) } \quad \hat{B}_{5}(n) & =\frac{5}{2} \sigma_{5}(n)-\frac{5}{2} \hat{\sigma}_{4}(n)-40 \sum_{m=1}^{n-1} \hat{\sigma}_{1}(m) \hat{\sigma}_{3}(n-m), \\
\text { (c) } \quad \hat{B}_{7}(n) & =\frac{7}{2} \sigma_{7}(n)-\frac{7}{2} \hat{\sigma}_{6}(n)-84 \sum_{m=1}^{n-1} \hat{\sigma}_{1}(m) \hat{\sigma}_{5}(n-m) \\
& -140 \sum_{m=1}^{n-1} \hat{\sigma}_{3}(m) \hat{\sigma}_{3}(n-m) .
\end{aligned}
$$

Lemma 3. ([4]) If $k \geq 1$ and $N \in N$, then

$$
\sum_{s=0}^{k-1}\left(\begin{array}{c}
2 k \\
2 s+1
\end{array}\right) \sum_{m=1}^{N} \hat{\sigma}_{2 k-2 s-1}(2 m-1) \hat{\sigma}_{2 s+1}(2 N-2 m+1)=\frac{1}{4} \sigma_{2 k+1}^{*}(2 N) .
$$

Proof of the Theorem 2. Using Theorem 1, we obtain

$$
\begin{aligned}
& \sum_{s=0}^{k-1}\left(\begin{array}{c}
2 k \\
2 s+1
\end{array}\right) \sum_{m=1}^{2 N-1} \hat{\sigma}_{2 k-2 s-1}(m) \hat{\sigma}_{2 s+1}(2 N-m) \\
& =\frac{1}{2}\left[\sigma_{2 k+1}(2 N)-\hat{\sigma}_{2 k}(2 N)\right]-\frac{1}{2 k+1} \hat{B}_{2 k+1}(2 N) .
\end{aligned}
$$

Using (2.3) and Lemma 3, we get

$$
\begin{aligned}
& \sum_{s=1}^{k-1}\left(\begin{array}{c}
2 k \\
2 s+1
\end{array}\right) \sum_{m=1}^{N-1} \hat{\sigma}_{2 k-2 s-1}(2 m) \hat{\sigma}_{2 s+1}(2 N-2 m) \\
& \quad=\frac{1}{4}\left[\sigma_{2 k+1}(2 N)+\sigma_{2 k+1}(N)\right]-\frac{1}{2} \hat{\sigma}_{2 k}(2 N)-\frac{1}{2 k+1} \hat{B}_{2 k+1}(2 N) .
\end{aligned}
$$

With (2.4) and Lemma 3, we get

$$
\sum_{s=1}^{k-1}\left(\begin{array}{c}
2 k \\
2 s+1
\end{array}\right) \sum_{m=1}^{2 N-1}(-1)^{m} \hat{\sigma}_{2 k-2 s-1}(m) \hat{\sigma}_{2 s+1}(2 N-m)
$$




$$
=\frac{1}{2} \sigma_{2 k+1}(N)-\frac{1}{2} \hat{\sigma}_{2 k}(2 N)-\frac{1}{2 k+1} \hat{B}_{2 k+1}(2 N) \text {. }
$$

Example 2. With $k=2,3,4$ in Theorem 2, we have

(a)

$$
\begin{aligned}
& 40 B_{3}(x) B_{3}(y)-6\left[B_{3}(x)+B_{3}(y)\right]=-12\left[x B_{5}(y)+y B_{5}(x)\right] \\
& +6\left[(x+y-1) B_{5}(x+y)+(x-y) B_{5}(y-x)\right]+5\left[B_{6}(y-x)+B_{6}(x+y)\right],
\end{aligned}
$$

(b)

$$
\begin{aligned}
& B_{3}(x) B_{5}(y)+B_{5}(x) B_{3}(y)=\frac{1}{16}\left[B_{8}(y-x)-B_{8}(x+y)\right]-\frac{1}{7}\left[x B_{7}(y)+y B_{7}(x)\right] \\
&+\frac{1}{14}\left[B_{7}(x)+B_{7}(y)+(x+y-1) B_{7}(x+y)+(x-y) B_{7}(y-x)\right],
\end{aligned}
$$

(c)

$$
\begin{aligned}
B_{3}(x) & B_{7}(y)+B_{7}(x) B_{3}(y)=\frac{3}{120}\left[B_{1} 0(y-x)-B_{1} 0(x+y)\right] \\
- & \frac{1}{12}\left[x B_{9}(y)+y B_{9}(x)\right]+\frac{1}{24}\left[B_{9}(x)+B_{9}(y)\right. \\
+ & \left.(x+y-1) B_{9}(x+y)+(x-y) B_{9}(y-x)\right]-\frac{21}{10} B_{5}(x) B_{5}(y) .
\end{aligned}
$$

Proof of the Corollary 1. Using Theorem 1 and Theorem 2, we obtain

$$
\begin{aligned}
& (2 k) \sum_{\substack{m=1 \\
m \text { odd }}}^{2 N-1} \hat{\sigma}_{2 k-1}(m) \hat{\sigma}(2 N-m) \\
& \quad=\frac{1}{2} \sigma_{2 k+1}(N)+\frac{1}{2} \sigma_{2 k+1}(2 N)-\hat{\sigma}_{2 k}(2 N)-\frac{2}{2 k+1} \hat{B}_{2 k+1}(2 N) .
\end{aligned}
$$

Replacing $2 k$ by $l$ in (2.5), we get proof of the corollary.

Proof of the Corollary 2. Let $\mathrm{m}$ is odd. Using Theorem 1 and Theorem 2, we obtain

$$
\begin{aligned}
& (2 k) \sum_{\substack{m=1 \\
m \text { odd }}}^{2 N-1} \hat{\sigma}_{2 k-1}(m) \hat{\sigma}(2 N-m) \\
& \quad+2 \sum_{s=1}^{k-1}\left(\begin{array}{c}
2 k \\
2 s+1
\end{array}\right) \sum_{\substack{m=1 \\
m \text { odd }}}^{2 N-1} \hat{\sigma}_{2 k-2 s-1}(m) \hat{\boldsymbol{\sigma}}_{2 s+1}(2 N-m) \\
& \quad=\frac{1}{2}\left[\sigma_{2 k+1}(2 N)-\sigma_{2 k+1}(N)\right] .
\end{aligned}
$$


It is clearly known that $\sigma_{k}^{*}(2 N)=\sigma_{k}(2 N)-\sigma_{k}(N)$ and $\sigma_{k}^{*}(2 N)=2^{k} \sigma_{k}^{*}(N)$. After we used it in (2.6), we get proof of the corollary.

Proof of the Theorem 3. Replacing $2 m$ by $m$ in Lemma 2, we get

$$
\begin{aligned}
\sum_{k=0}^{m}\left(\begin{array}{c}
2 m \\
2 k
\end{array}\right) & \frac{B_{2 k+1}(x) B_{2 m-2 k+1}(y)}{(2 k+1)(2 m-2 k+1)} \\
= & \frac{1}{2(2 m+1)}\left\{(x+y-1) B_{2 m+1}(x+y)+(x-y) B_{2 m+1}(y-x)\right\} \\
& +\frac{1}{2(2 m+2)}\left\{B_{2 m+2}(y-x)-B_{2 m+2}(x+y)\right\}
\end{aligned}
$$

Using $B_{k}(1-x)=(-1)^{k} B_{k}(x)$ with $k \geq 0$, we derive

$$
\begin{aligned}
\sum_{k=1}^{m-1}\left(\begin{array}{c}
2 m \\
2 k
\end{array}\right) & \frac{B_{2 k+1}(x) B_{2 m-2 k+1}(y)}{(2 k+1)(2 m-2 k+1)}=\frac{1}{4 m+4}\left\{B_{2 m+2}(y-x)-B_{2 m+2}(x+y)\right\} \\
& -\frac{1}{2 m+1}\left\{x B_{2 m+1}(y)+y B_{2 m+1}(x)\right\}+\frac{1}{4 m+2}\left\{B_{2 m+1}(x)+B_{2 m+1}(y)\right. \\
& \left.++(x+y-1) B_{2 m+1}(x+y)+(x-y) B_{2 m+1}(y-x)\right\} .
\end{aligned}
$$

Example 3. With $m=2,3,4$ in Theorem 3, we have

(a)

$$
\begin{aligned}
& 40 B_{3}(x) B_{3}(y)-6\left[B_{3}(x)+B_{3}(y)\right]=-12\left[x B_{5}(y)+y B_{5}(x)\right] \\
& +6\left[(x+y-1) B_{5}(x+y)+(x-y) B_{5}(y-x)\right]+5\left[B_{6}(y-x)+B_{6}(x+y)\right],
\end{aligned}
$$

(b)

$$
\begin{gathered}
B_{3}(x) B_{5}(y)+B_{5}(x) B_{3}(y)=\frac{1}{16}\left[B_{8}(y-x)-B_{8}(x+y)\right]-\frac{1}{7}\left[x B_{7}(y)+y B_{7}(x)\right] \\
+\frac{1}{14}\left[B_{7}(x)+B_{7}(y)+(x+y-1) B_{7}(x+y)+(x-y) B_{7}(y-x)\right]
\end{gathered}
$$

(c)

$$
\begin{aligned}
B_{3}(x) & B_{7}(y)+B_{7}(x) B_{3}(y)=\frac{3}{120}\left[B_{10}(y-x)-B_{10}(x+y)\right] \\
- & \frac{1}{12}\left[x B_{9}(y)+y B_{9}(x)\right]+\frac{1}{24}\left[B_{9}(x)+B_{9}(y)\right. \\
+ & \left.(x+y-1) B_{9}(x+y)+(x-y) B_{9}(y-x)\right]-\frac{21}{10} B_{5}(x) B_{5}(y) .
\end{aligned}
$$




\section{Divisor functions and linear combination of Bernoulli polynomials}

We consider the polynomial

$$
\sum_{\substack{1 \leq m \leq p-1 \\
1 \leq m^{\prime} \leq q-1 \\
a, b, c, d \text { odd } \\
a+b+c+d=2 l}}\left(\begin{array}{c}
2 l \\
a, b, c, d
\end{array}\right) \hat{\boldsymbol{\sigma}}_{a}(m) \hat{\boldsymbol{\sigma}}_{b}(p-m) \hat{\boldsymbol{\sigma}}_{c}\left(m^{\prime}\right) \hat{\boldsymbol{\sigma}}_{d}\left(q-m^{\prime}\right)
$$

once more. Let's call this polynomial $\mathfrak{C}$. Actually, the polynomials $\mathfrak{C}$ are combinations of Bernoulli polynomials and divisor functions.

Proof of the Theorem 4. We note that

$$
\begin{aligned}
& \left(\begin{array}{c}
2 m \\
2 k
\end{array}\right)\left(\begin{array}{c}
2 k \\
2 s+1
\end{array}\right)\left(\begin{array}{c}
2 m-2 k \\
2 s^{\prime}+1
\end{array}\right)=\frac{(2 m) !}{(2 s+1) !(2 k-2 s-1) !\left(2 s^{\prime}+1\right) !\left(2 m-2 k-2 s^{\prime}-1\right) !} \\
& \left(\begin{array}{c}
2 m \\
2 s+1,2 k-2 s-1,2 s^{\prime}+1,2 m-2 k-2 s^{\prime}-1
\end{array}\right)=\left(\begin{array}{c}
2 m \\
a, b, c, d
\end{array}\right) .
\end{aligned}
$$

By Theorem 1 and (3.1), we obtain

$$
\begin{aligned}
& \mathfrak{C}= \sum_{\substack{1 \leq m \leq p-1 \\
1 \leq m^{\prime} \leq q-1 \\
a, b, c, d \text { odd } \\
a+b+c+d=2 l}}\left(\begin{array}{c}
2 l \\
a, b, c, d
\end{array}\right) \hat{\sigma}_{a}(m) \hat{\sigma}_{b}(p-m) \hat{\sigma}_{c}\left(m^{\prime}\right) \hat{\sigma}_{d}\left(q-m^{\prime}\right) \\
&= \sum_{w=1}^{l-1}\left(\begin{array}{c}
2 l \\
2 w
\end{array}\right)\left[\sum_{s=0}^{w-1}\left(\begin{array}{c}
2 w \\
2 s+1
\end{array}\right) \sum_{m=1}^{p-1} \hat{\sigma}_{2 w-2 s-1}(m) \hat{\sigma}_{2 s+1}(p-m)\right] \\
& \times\left[\sum_{s^{\prime}=0}^{l-w-1}\left(\begin{array}{c}
2 l-2 w \\
2 s^{\prime}+1
\end{array}\right) \sum_{m^{\prime}=1}^{q-1} \hat{\sigma}_{2 l-2 w-2 s^{\prime}-1}\left(m^{\prime}\right) \hat{\sigma}_{2 s^{\prime}+1}\left(q-m^{\prime}\right)\right] \\
&=\sum_{w=1}^{l-1}\left(\begin{array}{c}
2 l \\
2 w
\end{array}\right)\left\{\frac{1}{2} \sigma_{2 w+1}(p)-\frac{1}{2} \sigma_{2 w}(p)+\sigma_{2 w}\left(\frac{p}{2}\right)-\sum_{d \mid p} \frac{B_{2 w+1}(d)}{2 w+1}\right. \\
&\left.+2 \sum_{\left.d\right|^{\frac{p}{2}}} \frac{B_{2 w+1}(d)}{2 w+1}\right\} \times\left\{\frac{1}{2} \sigma_{2 l-2 w+1}(q)-\frac{1}{2} \sigma_{2 l-2 w}(q)+\sigma_{2 l-2 w}\left(\frac{q}{2}\right)\right. \\
&\left.\quad-\sum_{d^{\prime} \mid q} \frac{B_{2 l-2 w+1}\left(d^{\prime}\right)}{2 l-2 w+1}+2 \sum_{d^{\prime} \mid \frac{q}{2}} \frac{B_{2 l-2 w+1}\left(d^{\prime}\right)}{2 l-2 w+1}\right\} .
\end{aligned}
$$

Consider the terms of $\mathfrak{C}$, we obtain

$$
\mathfrak{C}_{(1)}:=\frac{1}{4} \sum_{w=1}^{l-1}\left(\begin{array}{c}
2 l \\
2 w
\end{array}\right) \sigma_{2 w+1}(p) \sigma_{2 l-2 w+1}(q),
$$




$$
\begin{aligned}
& \mathfrak{C}_{(2)}:=-\frac{1}{4} \sum_{w=1}^{l-1}\left(\begin{array}{c}
2 l \\
2 w
\end{array}\right) \sigma_{2 w+1}(p) \sigma_{2 l-2 w}(q), \\
& \mathfrak{C}_{(3)}:=\frac{1}{2} \sum_{w=1}^{l-1}\left(\begin{array}{c}
2 l \\
2 w
\end{array}\right) \sigma_{2 w+1}(p) \sigma_{2 l-2 w}\left(\frac{q}{2}\right), \\
& \mathfrak{C}_{(4)}:=-\frac{1}{4} \sum_{w=1}^{l-1}\left(\begin{array}{c}
2 l \\
2 w
\end{array}\right) \sigma_{2 w}(p) \sigma_{2 l-2 w+1}(q), \\
& \mathfrak{C}_{(5)}:=\frac{1}{4} \sum_{w=1}^{l-1}\left(\begin{array}{c}
2 l \\
2 w
\end{array}\right) \sigma_{2 w}(p) \sigma_{2 l-2 w}(q), \\
& \mathfrak{C}_{(6)}:=-\frac{1}{2} \sum_{w=1}^{l-1}\left(\begin{array}{c}
2 l \\
2 w
\end{array}\right) \sigma_{2 w}(p) \sigma_{2 l-2 w}\left(\frac{q}{2}\right), \\
& \mathfrak{C}_{(7)}:=\frac{1}{2} \sum_{w=1}^{l-1}\left(\begin{array}{c}
2 l \\
2 w
\end{array}\right) \sigma_{2 w}\left(\frac{p}{2}\right) \sigma_{2 l-2 w+1}(q), \\
& \mathfrak{C}_{(8)}:=-\frac{1}{2} \sum_{w=1}^{l-1}\left(\begin{array}{c}
2 l \\
2 w
\end{array}\right) \sigma_{2 w}\left(\frac{p}{2}\right) \sigma_{2 l-2 w}(q), \\
& \mathfrak{C}_{(9)}:=\sum_{w=1}^{l-1}\left(\begin{array}{c}
2 l \\
2 w
\end{array}\right) \sigma_{2 w}\left(\frac{p}{2}\right) \sigma_{2 l-2 w}\left(\frac{q}{2}\right), \\
& \mathfrak{C}_{(10)}:=-\frac{1}{2} \sum_{d \mid p} \sum_{w=1}^{l-1}\left(\begin{array}{c}
2 l \\
2 w
\end{array}\right) \frac{B_{2 w+1}(d)}{2 w+1} \sigma_{2 l-2 w+1}(q), \\
& \mathfrak{C}_{(11)}:=\frac{1}{2} \sum_{d \mid p} \sum_{w=1}^{l-1}\left(\begin{array}{c}
2 l \\
2 w
\end{array}\right) \frac{B_{2 w+1}(d)}{2 w+1} \sigma_{2 l-2 w}(q), \\
& \mathfrak{C}_{(12)}:=-\sum_{d \mid p} \sum_{w=1}^{l-1}\left(\begin{array}{c}
2 l \\
2 w
\end{array}\right) \frac{B_{2 w+1}(d)}{2 w+1} \sigma_{2 l-2 w}\left(\frac{q}{2}\right), \\
& \mathfrak{C}_{(13)}:=-\frac{1}{2} \sum_{d^{\prime} \mid q} \sum_{w=1}^{l-1}\left(\begin{array}{c}
2 l \\
2 w
\end{array}\right) \frac{B_{2 l-2 w+1}\left(d^{\prime}\right)}{2 l-2 w+1} \sigma_{2 w+1}(p), \\
& \mathfrak{C}_{(14)}:=\frac{1}{2} \sum_{d^{\prime} \mid q} \sum_{w=1}^{l-1}\left(\begin{array}{c}
2 l \\
2 w
\end{array}\right) \frac{B_{2 l-2 w+1}\left(d^{\prime}\right)}{2 l-2 w+1} \sigma_{2 w}(p), \\
& \mathfrak{C}_{(15)}:=-\sum_{d^{\prime} \mid q} \sum_{w=1}^{l-1}\left(\begin{array}{c}
2 l \\
2 w
\end{array}\right) \frac{B_{2 l-2 w+1}\left(d^{\prime}\right)}{2 l-2 w+1} \sigma_{2 w}\left(\frac{p}{2}\right), \\
& \mathfrak{C}_{(16)}:=\sum_{d \mid \frac{p}{2}} \sum_{w=1}^{l-1}\left(\begin{array}{c}
2 l \\
2 w
\end{array}\right) \frac{B_{2 w+1}(d)}{2 w+1} \sigma_{2 l-2 w+1}(q),
\end{aligned}
$$




$$
\begin{aligned}
& \mathfrak{C}_{(17)}:=-\sum_{d \mid \frac{p}{2}} \sum_{w=1}^{l-1}\left(\begin{array}{c}
2 l \\
2 w
\end{array}\right) \frac{B_{2 w+1}(d)}{2 w+1} \sigma_{2 l-2 w}(q), \\
& \mathfrak{C}_{(18)}:=2 \sum_{d \mid \frac{p}{2}} \sum_{w=1}^{l-1}\left(\begin{array}{c}
2 l \\
2 w
\end{array}\right) \frac{B_{2 w+1}(d)}{2 w+1} \sigma_{2 l-2 w}\left(\frac{q}{2}\right), \\
& \mathfrak{C}_{(19)}:=\sum_{d^{\prime} \mid \frac{q}{2}} \sum_{w=1}^{l-1}\left(\begin{array}{c}
2 l \\
2 w
\end{array}\right) \frac{B_{2 l-2 w+1}\left(d^{\prime}\right)}{2 l-2 w+1} \sigma_{2 w+1}(p), \\
& \mathfrak{C}_{(20)}:=-\sum_{d^{\prime} \mid \frac{q}{2}} \sum_{w=1}^{l-1}\left(\begin{array}{c}
2 l \\
2 w
\end{array}\right) \frac{B_{2 l-2 w+1}\left(d^{\prime}\right)}{2 l-2 w+1} \sigma_{2 w}(p), \\
& \mathfrak{C}_{(21)}:=2 \sum_{d^{\prime} \mid \frac{q}{2}} \sum_{w=1}^{l-1}\left(\begin{array}{c}
2 l \\
2 w
\end{array}\right) \frac{B_{2 l-2 w+1}\left(d^{\prime}\right)}{2 l-2 w+1} \sigma_{2 w}\left(\frac{p}{2}\right), \\
& \mathfrak{C}_{(22)}:=\sum_{\substack{d\left|p \\
d^{\prime}\right| q}}^{l-1} \sum_{w=1}\left(\begin{array}{c}
2 l \\
2 w
\end{array}\right) \frac{B_{2 w+1}(d)}{2 w+1} \frac{B_{2 l-2 w+1}\left(d^{\prime}\right)}{2 l-2 w+1}, \\
& \mathfrak{C}_{(23)}:=4 \sum_{\substack{d\left|\frac{p}{2} \\
d^{\prime}\right| \frac{q}{2}}}^{l-1} \sum_{w=1}\left(\begin{array}{c}
2 l \\
2 w
\end{array}\right) \frac{B_{2 w+1}(d)}{2 w+1} \frac{B_{2 l-2 w+1}\left(d^{\prime}\right)}{2 l-2 w+1}, \\
& \mathfrak{C}_{(24)}:=-2 \sum_{\substack{d\left|\frac{p}{2} \\
d^{\prime}\right| q}}^{l-1} \sum_{w=1}\left(\begin{array}{c}
2 l \\
2 w
\end{array}\right) \frac{B_{2 w+1}(d)}{2 w+1} \frac{B_{2 l-2 w+1}\left(d^{\prime}\right)}{2 l-2 w+1},
\end{aligned}
$$

and

$$
\mathfrak{C}_{(25)}:=-2 \sum_{\substack{d\left|p \\
d^{\prime}\right| \frac{q}{2}}}^{l-1}\left(\begin{array}{c}
2 l \\
2 w
\end{array}\right) \frac{B_{2 w+1}(d)}{2 w+1} \frac{B_{2 l-2 w+1}\left(d^{\prime}\right)}{2 l-2 w+1} .
$$

From the binomial theorem,

$$
\sum_{w=1}^{l-1}\left(\begin{array}{c}
2 l \\
2 w
\end{array}\right) d^{\prime 2 l-2 w} d^{2 w}=\frac{1}{2}\left[\left(d^{\prime}+d\right)^{2 l}+\left(d^{\prime}-d\right)^{2 l}\right]-d^{2 l}-d^{2 l},
$$

we obtain

$$
\mathfrak{C}_{(1)}=\frac{1}{8} \sum_{\substack{d\left|p \\ d^{\prime}\right| q}} d^{\prime} d\left[\left(d^{\prime}+d\right)^{2 l}+\left(d^{\prime}-d\right)^{2 l}\right]-\frac{1}{4} \sum_{\substack{d\left|p \\ d^{\prime}\right| q}} d d^{2 l+1}-\frac{1}{4} \sum_{\substack{d\left|p \\ d^{\prime}\right| q}} d^{2 l+1} d^{\prime} .
$$

By the property of Bernoulli polynomial,

$$
B_{n}(x+1)-B_{n}(x)=n x^{n-1},
$$


we get

$$
\begin{aligned}
\mathfrak{C}_{(1)} & =\frac{1}{8(2 l+1)} \sum_{\substack{d\left|p \\
d^{\prime}\right| q}} d^{\prime} d\left[B_{2 l+1}\left(d^{\prime}+d+1\right)+B_{2 l+1}\left(d^{\prime}-d+1\right)\right. \\
& \left.-B_{2 l+1}\left(d^{\prime}+d\right)-B_{2 l+1}\left(d^{\prime}-d\right)\right]-\frac{1}{4}\left[\sigma_{1}(p) \sigma_{2 l+1}(q)+\sigma_{2 l+1}(p) \sigma_{1}(q)\right] .
\end{aligned}
$$

Similarly, we derive that

$$
\begin{array}{rl}
\mathfrak{C}_{(2)} & =-\frac{1}{8(2 l+1)} \sum_{\substack{d\left|p \\
d^{\prime}\right| q}} d\left[B_{2 l+1}\left(d^{\prime}+d+1\right)+B_{2 l+1}\left(d^{\prime}-d+1\right)-B_{2 l+1}\left(d^{\prime}+d\right)\right. \\
& \left.-B_{2 l+1}\left(d^{\prime}-d\right)\right]+\frac{1}{4}\left[\sigma_{1}(p) \sigma_{2 l}(q)+\sigma_{2 l+1}(p) \sigma_{0}(q)\right], \\
\mathfrak{C}_{(3)} & =\frac{1}{4(2 l+1)} \sum_{d \mid p} d\left[B_{2 l+1}\left(d^{\prime}+d+1\right)+B_{2 l+1}\left(d^{\prime}-d+1\right)-B_{2 l+1}\left(d^{\prime}+d\right)\right. \\
& \left.-B_{2 l+1}\left(d^{\prime}-d\right)\right]-\frac{1}{2}\left[\sigma_{1}(p) \sigma_{2 l}\left(\frac{q}{2}\right)+\sigma_{2 l+1}(p) \sigma_{0}\left(\frac{q}{2}\right)\right], \\
\mathfrak{C}_{(4)} & =-\frac{1}{8(2 l+1)} \sum_{d \mid p} d^{\prime}\left[B_{2 l+1}\left(d^{\prime}+d+1\right)+B_{2 l+1}\left(d^{\prime}-d+1\right)-B_{2 l+1}\left(d^{\prime}+d\right)\right. \\
& \left.-B_{2 l+1}\left(d^{\prime}-d\right)\right]+\frac{1}{4}\left[\sigma_{0}(p) \sigma_{2 l+1}(q)+\sigma_{2 l}(p) \sigma_{1}(q)\right], \\
\mathfrak{C}_{(5)} & =\frac{1}{8(2 l+1)} \sum_{d \mid p}\left[B_{2 l+1}\left(d^{\prime}+d+1\right)+B_{2 l+1}\left(d^{\prime}-d+1\right)\right. \\
& \left.-B_{2 l+1}\left(d^{\prime}+d\right)-B_{2 l+1}\left(d^{\prime}-d\right)\right]-\frac{1}{4}\left[\sigma_{0}(p) \sigma_{2 l}(q)+\sigma_{2 l}(p) \sigma_{0}(q)\right], \\
\mathfrak{C}_{(6)} & =-\frac{1}{4(2 l+1)} \sum_{d \mid p}\left[B_{2 l+1}\left(d^{\prime}+d+1\right)+B_{2 l+1}\left(d^{\prime}-d+1\right)-B_{2 l+1}\left(d^{\prime}+d\right)\right. \\
d^{\prime} \mid \frac{q}{2} & \left.-B_{2 l+1}\left(d^{\prime}-d\right)\right]+\frac{1}{2}\left[\sigma_{0}(p) \sigma_{2 l}\left(\frac{q}{2}\right)+\sigma_{2 l}(p) \sigma_{0}\left(\frac{q}{2}\right)\right], \\
\mathfrak{C}_{(7)}= & \frac{1}{4(2 l+1)} \sum_{d \mid \frac{p}{2}} d^{\prime}\left[B_{2 l+1}\left(d^{\prime}+d+1\right)+B_{2 l+1}\left(d^{\prime}-d+1\right)-B_{2 l+1}\left(d^{\prime}+d\right)\right. \\
d^{\prime} \mid q & 1 \\
&
\end{array}
$$




$$
\begin{aligned}
\mathfrak{C}_{(8)} & =-\frac{1}{4(2 l+1)} \sum_{\substack{d\left|\frac{p}{2} \\
d^{\prime}\right| q}}\left[B_{2 l+1}\left(d^{\prime}+d+1\right)+B_{2 l+1}\left(d^{\prime}-d+1\right)-B_{2 l+1}\left(d^{\prime}+d\right)\right. \\
& \left.-B_{2 l+1}\left(d^{\prime}-d\right)\right]+\frac{1}{2}\left[\sigma_{0}\left(\frac{p}{2}\right) \sigma_{2 l}(q)+\sigma_{2 l}\left(\frac{p}{2}\right) \sigma_{0}(q)\right], \\
\mathfrak{C}_{(9)} & =\frac{1}{2(2 l+1)} \sum_{\substack{d\left|\frac{p}{2} \\
d^{\prime}\right| \frac{q}{2}}}\left[B_{2 l+1}\left(d^{\prime}+d+1\right)+B_{2 l+1}\left(d^{\prime}-d+1\right)-B_{2 l+1}\left(d^{\prime}+d\right)\right. \\
& \left.-B_{2 l+1}\left(d^{\prime}-d\right)\right]-\left[\sigma_{0}\left(\frac{p}{2}\right) \sigma_{2 l}\left(\frac{q}{2}\right)+\sigma_{2 l}\left(\frac{p}{2}\right) \sigma_{0}\left(\frac{q}{2}\right)\right] .
\end{aligned}
$$

We obtain

$$
\begin{aligned}
\mathfrak{C}_{(10)} & =-\frac{1}{2} \sum_{w=1}^{l-1}\left(\begin{array}{c}
2 l \\
2 w
\end{array}\right) \sum_{d \mid p} \frac{B_{2 w+1}(d)}{2 w+1} \sigma_{2 l-2 w+1}(q) \\
& =-\frac{1}{2(2 l+1)} \sum_{w=1}^{l-1} \frac{(2 l+1)(2 l) !}{(2 w+1) !(2 l-2 w) !} \sum_{\substack{d\left|p \\
d^{\prime}\right| q}} B_{2 w+1}(d) \sigma_{2 l+1-2 w}(q) \\
& =-\frac{1}{4(2 l+1)} \sum_{d \mid p} d^{\prime}\left[B_{2 l+1}\left(d+d^{\prime}\right)+B_{2 l+1}\left(d-d^{\prime}\right)\right] \\
& +\frac{1}{2} \sigma_{1}(p) \sigma_{2 l+1}(q)-\frac{1}{4} \sigma_{0}(p) \sigma_{2 l+1}(q)+\frac{1}{2(2 l+1)} \sigma_{1}(q) \sum_{d \mid p} B_{2 l+1}(d)
\end{aligned}
$$

Similarly, like $\mathfrak{C}_{(10)}$, we get

$$
\begin{aligned}
\mathfrak{C}_{(11)} & =\frac{1}{4(2 l+1)} \sum_{\substack{d\left|p \\
d^{\prime}\right| q}}\left[B_{2 l+1}\left(d+d^{\prime}\right)+B_{2 l+1}\left(d-d^{\prime}\right)\right] \\
& -\frac{1}{2} \sigma_{1}(p) \sigma_{2 l}(q)+\frac{1}{4} \sigma_{0}(p) \sigma_{2 l}(q)-\frac{1}{2(2 l+1)} \sigma_{0}(q) \sum_{d \mid p} B_{2 l+1}(d), \\
\mathfrak{C}_{(12)} & =-\frac{1}{2(2 l+1)} \sum_{d \mid p}\left[B_{2 l+1}\left(d+d^{\prime}\right)+B_{2 l+1}\left(d-d^{\prime}\right)\right]+\sigma_{1}(p) \sigma_{2 l}\left(\frac{q}{2}\right) \\
& -\frac{1}{2} \sigma_{0}(p) \sigma_{2 l}\left(\frac{q}{2}\right)+\frac{1}{2 l+1} \sigma_{0}\left(\frac{q}{2}\right) \sum_{d \mid p} B_{2 l+1}(d), \\
\mathfrak{C}_{(13)}= & -\frac{1}{4(2 l+1)} \sum_{d \mid p} d\left[B_{2 l+1}\left(d^{\prime}+d\right)+B_{2 l+1}\left(d^{\prime}-d\right)\right]+\frac{1}{2} \sigma_{2 l+1}(p) \sigma_{1}(q)
\end{aligned}
$$




$$
\begin{aligned}
& -\frac{1}{4} \sigma_{2 l+1}(p) \sigma_{0}(q)+\frac{1}{2(2 l+1)} \sigma_{1}(p) \sum_{d^{\prime} \mid q} B_{2 l+1}\left(d^{\prime}\right), \\
& \mathfrak{C}_{(14)}=\frac{1}{4(2 l+1)} \sum_{\substack{d\left|p \\
d^{\prime}\right| q}}\left[B_{2 l+1}\left(d^{\prime}+d\right)+B_{2 l+1}\left(d^{\prime}-d\right)\right]-\frac{1}{2} \sigma_{2 l}(p) \sigma_{1}(q) \\
& +\frac{1}{4} \sigma_{2 l}(p) \sigma_{0}(q)-\frac{1}{2(2 l+1)} \sigma_{0}(p) \sum_{d^{\prime} \mid q} B_{2 l+1}\left(d^{\prime}\right), \\
& \mathfrak{C}_{(15)}=-\frac{1}{2(2 l+1)} \sum_{\substack{\left.d\right|^{p} \\
d^{\prime} \mid q}}\left[B_{2 l+1}\left(d^{\prime}+d\right)+B_{2 l+1}\left(d^{\prime}-d\right)\right]+\sigma_{2 l}\left(\frac{p}{2}\right) \sigma_{1}(q) \\
& -\frac{1}{2} \sigma_{2 l}\left(\frac{p}{2}\right) \sigma_{0}(q)+\frac{1}{2 l+1} \sigma_{0}\left(\frac{p}{2}\right) \sum_{d^{\prime} \mid q} B_{2 l+1}\left(d^{\prime}\right), \\
& \mathfrak{C}_{(16)}=\frac{1}{2(2 l+1)} \sum_{\substack{d\left|\frac{p}{2} \\
d^{\prime}\right| q}} d^{\prime}\left[B_{2 l+1}\left(d^{\prime}+d\right)+B_{2 l+1}\left(d-d^{\prime}\right)\right]-\sigma_{1}\left(\frac{p}{2}\right) \sigma_{2 l+1}(q) \\
& +\frac{1}{2} \sigma_{0}\left(\frac{p}{2}\right) \sigma_{2 l+1}(q)-\frac{1}{2 l+1} \sigma_{1}(q) \sum_{d \mid \frac{p}{2}} B_{2 l+1}(d), \\
& \mathfrak{C}_{(17)}=-\frac{1}{2(2 l+1)} \sum_{\substack{\left.d\right|^{\frac{p}{2}} \\
d^{\prime} \mid q}}\left[B_{2 l+1}\left(d+d^{\prime}\right)+B_{2 l+1}\left(d-d^{\prime}\right)\right]+\sigma_{1}\left(\frac{p}{2}\right) \sigma_{2 l}(q) \\
& -\frac{1}{2} \sigma_{0}\left(\frac{p}{2}\right) \sigma_{2 l}(q)+\frac{1}{2 l+1} \sigma_{0}(q) \sum_{d \mid \frac{p}{2}} B_{2 l+1}(d), \\
& \mathfrak{C}_{(18)}=\frac{1}{2 l+1} \sum_{\substack{d\left|\frac{p}{2} \\
d^{\prime}\right| \frac{q}{2}}}\left[B_{2 l+1}\left(d+d^{\prime}\right)+B_{2 l+1}\left(d-d^{\prime}\right)\right]-2 \sigma_{1}\left(\frac{p}{2}\right) \sigma_{2 l}\left(\frac{q}{2}\right) \\
& +\sigma_{0}\left(\frac{p}{2}\right) \sigma_{2 l}\left(\frac{q}{2}\right)-\frac{2}{2 l+1} \sigma_{0}\left(\frac{q}{2}\right) \sum_{d \mid \frac{p}{2}} B_{2 l+1}(d), \\
& \mathfrak{C}_{(19)}=\frac{1}{2(2 l+1)} \sum_{d \mid p} d\left[B_{2 l+1}\left(d^{\prime}+d\right)+B_{2 l+1}\left(d^{\prime}-d\right)\right]-\sigma_{2 l+1}(p) \sigma_{1}\left(\frac{q}{2}\right) \\
& +\frac{1}{2} \sigma_{2 l+1}(p) \sigma_{0}\left(\frac{q}{2}\right)-\frac{1}{2 l+1} \sigma_{1}(p) \sum_{d^{\prime} \mid \frac{q}{2}} B_{2 l+1}\left(d^{\prime}\right)
\end{aligned}
$$




$$
\begin{aligned}
\mathfrak{C}_{(20)} & =-\frac{1}{2(2 l+1)} \sum_{\substack{d\left|p \\
d^{\prime}\right| \frac{q}{2}}}\left[B_{2 l+1}\left(d^{\prime}+d\right)+B_{2 l+1}\left(d^{\prime}-d\right)\right]+\sigma_{2 l}(p) \sigma_{1}\left(\frac{q}{2}\right) \\
& -\frac{1}{2} \sigma_{2 l}(p) \sigma_{0}\left(\frac{q}{2}\right)+\frac{1}{2 l+1} \sigma_{0}(p) \sum_{d^{\prime} \mid \frac{q}{2}} B_{2 l+1}\left(d^{\prime}\right)
\end{aligned}
$$

and

$$
\begin{aligned}
\mathfrak{C}_{(21)} & =\frac{1}{2 l+1} \sum_{\substack{d\left|\frac{p}{2} \\
d^{\prime}\right| \frac{q}{2}}}\left[B_{2 l+1}\left(d^{\prime}+d\right)+B_{2 l+1}\left(d^{\prime}-d\right)\right]-2 \sigma_{2 l}\left(\frac{p}{2}\right) \sigma_{1}\left(\frac{q}{2}\right) \\
& +\sigma_{2 l}\left(\frac{p}{2}\right) \sigma_{0}\left(\frac{q}{2}\right)-\frac{2}{2 l+1} \sigma_{0}\left(\frac{p}{2}\right) \sum_{d^{\prime} \mid \frac{q}{2}} B_{2 l+1}\left(d^{\prime}\right) .
\end{aligned}
$$

From Lemma 2, then we get

$$
\begin{aligned}
\mathfrak{C}_{(22)} & =\sum_{\substack{d\left|p \\
d^{\prime}\right| q}}^{l-1} \sum_{w=1}\left(\begin{array}{c}
2 l \\
2 w
\end{array}\right) \frac{B_{2 w+1}(d)}{2 w+1} \frac{B_{2 l-2 w+1}\left(d^{\prime}\right)}{2 l-2 w+1} \\
& =\frac{1}{2(2 l+1)} \sum_{d \mid p}\left[\left(d+d^{\prime}-1\right) B_{2 l+1}\left(d+d^{\prime}\right)+\left(d-d^{\prime}\right) B_{2 l+1}\left(d^{\prime}-d\right)\right. \\
& \left.-(2 d-1) B_{2 l+1}\left(d^{\prime}\right)-\left(2 d^{\prime}-1\right) B_{2 l+1}(d)\right] \\
& -\frac{1}{4(l+1)} \sum_{\substack{d\left|p \\
d^{\prime}\right| q}}\left[B_{2 l+2}\left(d+d^{\prime}\right)-B_{2 l+2}\left(d^{\prime}-d\right)\right], \\
\mathfrak{C}_{(23)} & =\frac{4}{2(2 l+1)} \sum_{d \mid \frac{p}{2}}\left[\left(d+d^{\prime}-1\right) B_{2 l+1}\left(d+d^{\prime}\right)+\left(d-d^{\prime}\right) B_{2 l+1}\left(d^{\prime}-d\right)\right. \\
& \left.-(2 d-1) B_{2 l+1}\left(d^{\prime}\right)-\left(2 d^{\prime}-1\right) B_{2 l+1}(d)\right] \\
& -\frac{4}{4(l+1)} \sum_{\substack{d^{\prime}\left|\frac{p}{2} \\
d^{\prime}\right| \frac{q}{2}}}\left[B_{2 l+2}\left(d+d^{\prime}\right)-B_{2 l+2}\left(d^{\prime}-d\right)\right], \\
\mathfrak{C}_{(24)} & =-\frac{2}{2(2 l+1)} \sum_{\substack{d^{\prime}\left|\frac{p}{2} \\
d^{\prime}\right| q}}\left[\left(d+d^{\prime}-1\right) B_{2 l+1}\left(d+d^{\prime}\right)+\left(d-d^{\prime}\right) B_{2 l+1}\left(d^{\prime}-d\right)\right. \\
& \left.-(2 d-1) B_{2 l+1}\left(d^{\prime}\right)-\left(2 d^{\prime}-1\right) B_{2 l+1}(d)\right]
\end{aligned}
$$




$$
\begin{aligned}
& +\frac{2}{4(l+1)} \sum_{\substack{d\left|\frac{p}{2} \\
d^{\prime}\right| q}}\left[B_{2 l+2}\left(d+d^{\prime}\right)-B_{2 l+2}\left(d^{\prime}-d\right)\right] \\
\mathfrak{C}_{(25)} & =-\frac{2}{2(2 l+1)} \sum_{\substack{d\left|p \\
d^{\prime}\right| \frac{q}{2}}}\left[\left(d+d^{\prime}-1\right) B_{2 l+1}\left(d+d^{\prime}\right)+\left(d-d^{\prime}\right) B_{2 l+1}\left(d^{\prime}-d\right)\right. \\
& \left.-(2 d-1) B_{2 l+1}\left(d^{\prime}\right)-\left(2 d^{\prime}-1\right) B_{2 l+1}(d)\right] \\
& +\frac{2}{4(l+1)} \sum_{\substack{d\left|p \\
d^{\prime}\right| \frac{q}{2}}}\left[B_{2 l+2}\left(d+d^{\prime}\right)-B_{2 l+2}\left(d^{\prime}-d\right)\right]
\end{aligned}
$$

Summing $\mathfrak{C}_{(i)}(i=1, \ldots, 25)$, we drive the Theorem.

Example 4. In Theorem 4, for $l=a=b=c=d=1$ and $p=q=2$, we get

$$
\sum_{\substack{1 \leq m \leq p-1 \\
1 \leq m^{\prime} \leq q-1 \\
a, b, c, d \text { odd } \\
a+b+c+d=2 l}}\left(\begin{array}{c}
2 l \\
a, b, c, d
\end{array}\right) \hat{\sigma}_{a}(m) \hat{\sigma}_{b}(p-m) \hat{\sigma}_{c}\left(m^{\prime}\right) \hat{\sigma}_{d}\left(q-m^{\prime}\right)=4 !
$$

\section{REFERENCES}

[1] H. P. B. Cho, D. Kim, "Evaluation of a certain combinatorial convolution sum in higher level cases." J. Math. Anal. Appl., vol. 406, no. 1, pp. 203-210, 2013, doi: https://doi.org/10.1016/j.jmaa.2013.04.052.

[2] B. C. Berndt, Ramanujan's Notebooks, Part II. New York: Springer-Verlag, $1989 . \quad$ doi: 10.1007/978-1-4612-4530-8, 978-1-4612-4530-8.

[3] M. Besge, "Extrait d'une lettre de M. Besge à M. Liouville.” J. Math. Pures Appl., vol. 21, no. 2, pp. 423-441, 2007, doi: 10.1137/050641867.

[4] A. B. D. Kim, "Convolution identities for twisted Eisenstein series and twisted divisor functions." Fixed Point Theory and Applications, vol. 81, 2013, doi: https://doi.org/10.1186/1687-1812-201381.

[5] N. I. D. Kim, "Certain combinatoric Bernoulli polynomials and convolution sums of divisor functions." Advance Difference Equations, vol. 2013, no. 310, 2013.

[6] H. Hahn, "Convolution sums of some functions on divisors." Rocky Mountain Journal of Mathematics, vol. 37, no. 5, pp. 1593-1622, 2007.

[7] A. P. H.M. Srivastava, "Remarks on some relationships between the Bernoulli and Euler polynomials." Applied Mathematics Letters, vol. 17, pp. 375-380, 2004.

[8] R. Z. W. Chu, "Convolutions of Bernoulli and Euler polynomials." Sarajevo Journal of Mathematics, vol. 6, no. 6(18), pp. 147-163, 2010.

[9] K. Williams, Number Theory in the Spirit of Liouville. Cambridge University Press, New York: Cambridge University Press, 2011. doi: https://doi.org/10.1017/CBO9780511751684. 
Authors' addresses

\section{Daeyeoul Kim}

Jeonbuk National University, Institute of Pure and Applied Mathematics, Department of Mathematics, 567 Baekje-daero, Deokjin-gu, Jeonju-si, Jeollabuk-do 54896, Republic of Korea

E-mail address: kdaeyeoul@ jbnu. ac.kr

Nazli Yildiz Ikikardes

(Corresponding author) Balikesir University, Necatibey Faculty of Education, Department of Mathematics and Science Education, 10100 Balikesir, Turkey

E-mail address: nyildiz@balikesir.edu.tr, nyildizikikardes@gmail.com 